\title{
Robust Topology Optimization under Load and Geometry Uncertainties by Using New Sparse Grid Collocation Method
}

\author{
Seyyed Ali Latifi Rostami ${ }^{1}$, Ali Ghoddosian ${ }^{1 *}$ \\ ${ }^{1}$ Department of Mechanical Engineering, \\ Faculty of Engineering, \\ Semnan University, P.O.B: 35131-19111, Semnan, Iran \\ * Corresponding author, e-mail: aghoddosian@semnan.ac.ir
}

Received: 03 September 2018, Accepted: 01 July 2019, Published online: 06 September 2019

\begin{abstract}
In this paper, a robust topology optimization method presents that insensitive to the uncertainty in geometry and applied load. Geometric uncertainty can be introduced in the manufacturing variability. Applied load uncertainty is occurring in magnitude and angle of force. These uncertainties can be modeled as a random field. A memory-less transformation of random fields used to random variation modeling. The Adaptive Sparse Grid Collocation (ASGC) method combined with the uncertainty models provides robust designs by utilizing already developed deterministic solvers. The proposed algorithm provides a computationally cheap alternative to previously introduced stochastic optimization methods based on Monte Carlo sampling by using the adaptive sparse grid method. Numerical examples, such as a 2D simply supported beam and cantilever beam as benchmark problems, are used to show the effectiveness and superiority of the ASGC method.
\end{abstract}

Keywords

topology optimization, load uncertainty, geometric uncertainty, sparse grid, collocation method

\section{Introduction}

Improving the performance and reducing the costs in engineering design of structures are obtained by using of structural optimization. Generally, size, shape and topology optimization are three levels of structural optimization. The purpose of the classic topology optimization is to obtain an optimal distribution of material or structural design parameters in a range of nominal material properties, geometry and loading conditions. SIMP and ESO methods are very popular in topology optimization and used in many articles. In these methods, elemental design variables are used to create topology optimization formulations.

Traditionally, the structural topology optimization is done deterministically (deterministic topology optimization (DET)) [1]. However, designs derived by deterministic approaches often tend to be sensitive to changes in system and operating parameters. To cope with this issue, safety factors have traditionally been introduced into the formulation of the design optimization problem, which often leads to unknowable unsafe or overly conservative designs. Therefore, a serious need to investigate the effect of uncertainty in the design of a structural topology is desirable.
A semi-definite programming approach for topology optimization of truss structure under uncertain load was presented by Ben-Tal and Nemirovski [2]. Guest and Igusa [3] considered load uncertainty in topology optimization. They consider the size and location of applied loads as uncertain. The weighted average multiple load pattern method has been developed in their research to resolve the problem of loading uncertainty.

Kogiso et al. [4] considered uncertainty in the applied load. Uncertainty in the direction of the load was supposed and optimization of the complaint mechanisms was determined. Variations were studied based on the sensitivity with the evaluated variance by using the first derivative. Dunning et al. [5], Cherkaev and Cherkaev [6], Logo [7] and Logo et al. [8], Zhao and Wang [9], Zhao et al. [10], were also studied robust topology optimization under uncertainty loading.

Chen et al. [11] presented a robust method for minimum compliance and complaint mechanisms by using the level set method. In their work uncertainties in loading and material properties have been studied. Modeling 
of random field by using of the spectral stochastic finite element method (SSFEM) is a novelty of their approach. Tootkaboni et al. [12] research on the robust shape and topology optimization of two-dimensional structures. They used a polynomial chaos approach for mass minimization of structure under material uncertainty.

More recently some researchers have been focused on modeling geometric uncertainty in topology and shape optimization by using both level set and density based methods [11, 13 and 14]. In density based topology optimization these uncertainties that are attributed to manufacturing tolerances are commonly modeled via the Heaviside thresholding approach [15-18].

Chen and Chen [13] considered geometric uncertainties by using level-set approach. They have presented a robust design optimization (RDO) for topology optimization. In their work a stochastic velocity field is modeled by using Hamilton-Jacobi equations. Then, random geometry variations are modeled through velocity field. The mean and standard deviation of the design criteria are evaluated using an efficient quadrature rule.

Geometric uncertainty is modeled in Lazarov et al. [17] by using the stochastic perturbation method. In their work, it is assumed that the system parameters, the inputs and the solution have small random variation. The method provides computationally cheap alternative to $\mathrm{MC}$, however, the need for small variability is a restriction for the use of this method on the general applicability.

Keshavarzzadeh et al. [19] considered topology optimization under uncertainty in load and geometry. They used non-intrusive polynomial chaos expansion with design sensitivity analysis for reliability-based and robust topology optimization. Manufacturing variability is modeled by using of thresholding technique. In this method, a reduced dimensional random field was used to demonstrate the threshold field.

Latifi Rostami and Ghoddosian [20] used adaptive sparse grid collocation method to study the topology optimization for mechanical systems with hybrid material and geometric uncertainties. The random variations are modeled by a memory-less transformation of random fields which ensures their physical admissibility. The computational cost is decreased by using of sparse grids and discretization refinement that are proposed and demonstrated as well. This method provides a computationally cheap alternative to previously introduced stochastic optimization methods based on Monte Carlo sampling by using the adaptive sparse grids method.
This paper organized as follows. First the deterministic (DET) optimization problem and then the Stochastic optimization approach for obtaining robust designs are presented. In the next section, modeling of uncertainties is discussed. Then, Adaptive Sparse Grid Collocation methods and its features are introduced. The optimization algorithm is presented in following and finally its applicability in topology optimization of robust minimum compliance is demonstrated.

\section{Problem definition}

One of the issues that are commonly discussed in topology optimization is the minimum compliance optimization. The formulation of this field is given as

$$
\begin{aligned}
\min _{\rho}: & f(\rho)=\mathbf{f}^{\mathbf{T}} \mathbf{u} \\
\text { s.t. } & : \mathbf{K u}=\mathbf{f} \\
& : V(\rho)=\sum_{i=1}^{N_{e}} \overline{\tilde{\rho}}_{i} v_{i} \leq V^{*} \\
& : 0 \leq \rho_{i} \leq 1 \quad \forall i \in N_{e},
\end{aligned}
$$

where $\mathbf{K}$ is the stiffness matrix obtained by finite element discretization, $\mathbf{u}$ and $\mathbf{f}$ is the solution and the input vectors for the system $N_{e}$ are the set of all elements and $\tilde{\bar{p}}$ are the physical density associated with. $V_{i}$ is a volume of element $i$ and $V^{*}$ is the fraction of the total volume which can be occupied with the material. The individual elements contributions to the tangent matrix $\mathbf{K}$ are calculated as $\mathbf{K}_{i}=E_{i} \mathbf{K}_{0}$ where $\mathbf{K}_{0}$ is the element stiffness matrix for unit stiffness and $E_{i}$ is the material stiffness obtained by using the so-called solid isotropic material interpolation with penalization (SIMP) given as

$E_{i}=E_{\min }+\overline{\tilde{\rho}}_{i}^{p}\left(E_{0}-E_{\min }\right)$,

where $E_{0}$ indicates the stiffness of places that occupied with the material, $p$ is the penalization factor and $\tilde{\bar{p}}_{i}$ is the physical density of element $i$. The vector $\mathbf{l}$ in Eq. (1) will have different values for different problems. In the test case that discussed minimum compliance design, $\mathbf{l}=\mathbf{f}$.

Problems governed by the mechanical stiffness suffer from numerical instabilities, such as checkerboard patterns and mesh dependency solution. Density filtering makes it possible to achieve independent designs of the mesh.

Here the mesh independent density filtering (Bruns and Tortorelli [21]) is used as a basis to ensure the existence of solutions. The basic idea is to determine the physical element density to be a weighted average of the neighboring design variables, where the neighborhood is defined by a circle in $2 \mathrm{D}$ or sphere in $3 \mathrm{D}$ with the specified radius. 
Applying regularization to the original problem causes gray areas with a moderate density of 0 to 1 .

In the following, the filtered density is denoted with $\tilde{p}_{i}$ and physical density with $\tilde{\bar{p}}_{i}$. The filtered density for $i$-th element is calculated as

$\tilde{\rho}_{i}=\frac{\sum_{j \in N_{e, i}} w\left(\mathbf{x}_{j}\right) \nu_{j} \rho_{j}}{\sum_{j \in N_{e, i}} w\left(\mathbf{x}_{j}\right) \nu_{j}}$,

where the neighborhood set of elements, locating within the filter domain for the element $i$ am represented by, the weighting function $w(\mathbf{x})$ is defined as

$w\left(\mathbf{x}_{j}\right)=R-\left|\mathbf{x}_{j}-\mathbf{x}_{i}\right|$.

In the above relation, $R$ the specified filter radius and $\mathbf{x}_{i}$ and $\mathbf{x}_{j}$ are central coordinates of the design elements $i$ and $\mathrm{j}$ respectively. The sensitivity of filtered density $\tilde{p}_{i}$ with respect to the design variables is calculated as

$$
\frac{\partial \tilde{\rho}_{i}}{\partial \rho_{i}}=\frac{w\left(\mathbf{x}_{j}\right) v_{j}}{\sum_{j \in N_{e, i}} w\left(\mathbf{x}_{j}\right) v_{j}} .
$$

However, if the physical density is shown by the filtered density, the design of the gray areas will be formed, which will be difficult to interpret. Projection schemes should be used to convert these gray areas to white and black areas. In this research, a Heaviside projection procedure is used for projecting these gray areas. In this method firstly a threshold $\eta$ is defined and then all values below threshold are projected for 0 and above the threshold to 1 . As the Heaviside function is not differentiable, it is approximated by a smooth approximation controlled by relaxation parameter $\beta$. A Heaviside projection utilized here is given as

$\overline{\tilde{\rho}}_{i}=\frac{\tanh (\beta \eta)+\tanh \left(\beta\left(\tilde{\rho}_{i}-\eta\right)\right)}{\tanh (\beta \eta)+\tanh (\beta(1-\eta))}$.

The derivative of the physical density $\tilde{\bar{p}}_{i}$ with respect to the filtered density $\tilde{p}_{i}$ is calculated as

$\frac{\partial \overline{\tilde{\rho}}_{i}}{\partial \tilde{\rho}_{i}}=\frac{\beta\left(\operatorname{sech}\left(\beta\left(\tilde{\rho}_{i}-\eta\right)\right)\right)^{2}}{\tanh (\beta \eta)+\tanh (\beta(1-\eta))}$.

The physical density $\tilde{\overline{p_{i}}}$ is a function of the filtered density $\tilde{p}_{i}$ and the sensitivities of the objective function in Eq. (1) with respect to the original design variables are calculated as follows (Eq. (8)):

$$
\frac{\partial f}{\partial \rho_{j}}=\sum_{i \in N_{e, j}} \frac{\partial f}{\partial \overline{\tilde{\rho}}_{i}} \frac{\partial \overline{\tilde{\rho}}_{i}}{\partial \tilde{\rho}_{i}} \frac{\partial \tilde{\rho}_{i}}{\partial \rho_{j}} .
$$

\section{Stochastic optimization}

When a stochastic system is discussed, its properties such as excitations, material or manufacturing errors will have a random nature (depending on the type of problem). Therefore the response $\mathbf{u}$ becomes a stochastic field and the objective in Eq. (1) becomes a random variable. The stochastic compliance objective function in robust form is commonly determined by using of the mean and standard deviation of the compliance in the form of the weighted sum.

$$
\begin{gathered}
\min _{\rho}: f(\rho)=\mathrm{E}[C]+\kappa \sqrt{\operatorname{Var}[C]} \\
\quad=\mathrm{E}\left[\mathbf{l}^{\mathrm{T}} \mathbf{u}\right]+\kappa \sqrt{\operatorname{Var}\left[\mathbf{l}^{\mathrm{T}} \mathbf{u}\right]} \\
\text { s.t. }: \mathbf{K u}=\mathbf{f} \\
\quad: \mathrm{E}[V(\rho)] \leq V^{*} \\
\quad: 0 \leq \rho_{i} \leq 1 \quad \forall i \in N_{e},
\end{gathered}
$$

where $\mathrm{E}[C]$ is the expected value of the compliance, the variance of the compliance is shown with $\operatorname{Var}[C]$ and represented the weighting coefficient that chosen by the user. In deterministic loading condition, the mean value (Expectancy) and the variance of the compliance of are given by

$$
\begin{aligned}
& E[C]=E\left[\mathbf{f}^{\mathrm{T}} \mathbf{u}\right]=\mathbf{f}_{0}^{\mathrm{T}} E[\mathbf{u}], \\
& \operatorname{Var}[C]=\operatorname{Var}\left[\mathbf{f}^{\mathrm{T}} \mathbf{u}\right] .
\end{aligned}
$$

The sensitivities of the objective function with respect to the design variables $\rho$ are found by using the adjoint method as follows (Eq. (12)):

$$
\frac{\partial f}{\partial \boldsymbol{\rho}}=\frac{\partial E[C]}{\partial \boldsymbol{\rho}}+\kappa \frac{\partial(\sqrt{\mathrm{V} \operatorname{ar}[C]})}{\partial \boldsymbol{\rho}} .
$$

In the following sections first, the representation of the uncertainties as a stochastic field is discussed and then the solution of the stochastic state problem by using the Probabilistic collocation method is presented in more details.

\section{Applied load and geometric uncertainty representation}

Under real-world conditions, many of the terms in the optimization problem, including applied loads, the stiffness of the structure and geometry may involve some degree of uncertainty. There exist a variety of uncertainty quantification schemes that can be used for design optimization under uncertainty. Depending on the amount of available information, uncertainties can be introduced in various ways in the analysis of physical systems. 
Commonly, probabilistic models used to quantify uncertainties. One or several system parameters, as well as system inputs are represented as stochastic processes in time or stochastic fields in space. Here, with the help of a scalar stochastic field $y(\mathbf{x}, \omega)$ the system properties and the geometric uncertainties are modeled. The Scalar stochastic field is defined in the physical domain $D \subset R^{d}$, and in this system $\Omega$ represent the set of all possible outcomes. $\omega \in \Omega$ is a coordinate which belong to it. Usually, the index set $D$ and the possible outcomes set $\Omega$ are infinite dimensional object. Therefore, any practical application would require to the reduction of dimensionality. In this research a truncated KLE used to model the reduced random field. The KLE provides a mapping from a relatively small number of independent random variables to the types of random fields that are common in many physical processes. The Random field should have the optimal range for topology optimization, in this research values between 0 and 1 , but $Z$ as a result of KLE does not in the range. Therefore, to resolve this problem an inverse transform sampling is applied to $Z$ to ensure the new random variable $(\hat{Z})$ in the optimal range.

To obtain the KLE expansion for the random field $Z$ its correlation function is assumed to be of the squared exponential form i.e.

$$
\mathrm{R}_{x x^{\prime}}=\exp \left(-\frac{d^{2}}{2 l_{c}^{2}}\right),
$$

where $d=\left|x-x^{\prime}\right|$ is the Euclidean distance between locations $x$ and $x^{\prime}$ and $l_{c}$ is the correlation length. The correlation length determines the scale of variation of the random field: a random field with a small correlation length will exhibit more fine scale variations than a random field with a large correlation length. A correlation length $l_{c}=0.3 \mathrm{~L}$ is used [22]. The correlation matrix $\mathrm{R}$ is obtained by the finite element centroids $x$ and $x^{\prime}$. The KL decomposition of a zero mean process generates by using the $n$ eigenvalue-eigenvector pairs $\left(\lambda_{i}, \gamma_{i}\right)$ of the correlation matrix as follows (Eq. (14):

$$
Z(x, \xi)=\sum_{i=1}^{n} \sqrt{\lambda_{i}} \gamma_{i}(x) \varphi_{i}(\xi) \approx \sum_{i=1}^{n_{\text {mode }}} \sqrt{\lambda_{i}} \gamma_{i}(x) \varphi_{i}(\xi),
$$

where random variables represented by $\varphi_{i}$ and $\gamma_{i}(x)$ is interpolated from the eigenvector $\gamma_{i}$ as piecewise uniform over the elements. For the purpose of dimension reduction, the expansion is truncated to the first $\mathrm{n}_{\text {mode }}<\mathrm{n}$ modes. The correlation length $l_{c}$ and the type of correlation function are effective in determining the number of modes $n_{\text {mode }}$.
Ultimately, the random field $Z$ generates by using the random variables $\varphi_{i}$ via Eq. (14). To that end, uniform random variables assign to each $\varphi_{i}$ with zero mean and unit variance. This assumption ensures that the same correlation matrix $\mathbf{R}$ can be reconstructed from the random field $Z$ of Eq. (14).

\subsection{Modeling uncertainty in applied load}

Loading uncertainties of linear elastic structures are considered in this section. Due to the variation of uncertain parameters $\xi$, the performance function $f(x, \xi)$ has a probability distribution description. Thus, the robustness of the design objective can be achieved by optimizing the mean performance and minimizing the standard deviation of the performance simultaneously.

In this research a random load with uncertain angle and magnitude is considered. These uncertainties are characterized by two independent random variables. Random angle supposed that has a uniform distribution. Therefore, this random field showed via Eq. (14). For magnitude, uncertainty is shown with Gaussian distribution with a mean of 1 and standard deviation of $0.3(F=N(1,0.3))$. Thus

$$
\begin{aligned}
& f(x, \xi)=F A(x, \xi) \\
& F=N(1,0.3), \quad A(x, \xi)=\sum_{i=1}^{n_{\text {mode }}} \sqrt{\lambda_{i}} \gamma_{i}(x) \varphi_{i}(\xi) .
\end{aligned}
$$

\subsection{Manufacturing uncertainty via random field}

We now consider geometric uncertainty in the above optimization problem. As mentioned above, geometric uncertainties are modeled by the assumption of a random nature to the threshold parameter $\eta$. This approach is model uncertainty in structures that are fabricated via etching. The etching process causes errors in the form of over- or underetching which produces structures that are either thinner or thicker than intended. This uncertainty is modelled with the Heaviside threshold. A non-uniform variation of errors in the design domain can be caused by the etching assumption, a more realistic. In the present paper this variation represents by $\eta$ with the random field such that:

$\eta,(x, \xi)=\alpha_{1} \hat{Z}(x, \xi)+\alpha_{2}$,

where $\hat{Z}(x, \xi) \in[0,1]$ is a random field, $\alpha_{1}$ and $\alpha_{2}$ control the mean and range of the process $\eta$ such that $\eta(x, \xi) \in[0,1]$.

Unfortunately realization of the $\operatorname{KLE} Z(x, \xi) \notin[0,1]$ and this is noteworthy. Indeed geometric uncertainty is introduced by randomly varying the threshold parameter $\eta$ and require $\eta(x, \xi) \in[0,1]$. To generate the random field $\hat{Z}$ of Eq. (13) such that $\hat{Z}(x, \xi) \in[0,1]$ use the fact that the Cumulative Density Function (CDF) of any continuous 
random variable, e.g. $Z(x, \xi)$ is a uniform random variable, ranging from 0 to 1 i.e. $U[0,1]$; this is ideal for modeling thresholds. In other words, for every realization of $Z(x, \xi)$ there is a unique CDF transformed value that belongs to the range $[0,1]$. The ensemble of these CDF transformed values has a uniform distribution from which the transformed process $\hat{Z}$ is defined such that

$\hat{\mathrm{Z}}(x, \xi)=C D F(Z(x, \xi))$.

\section{Adaptive sparse grid collocation methods}

\subsection{Sparse grid}

The sparse grid method is a most popular method that very useful in multidimensional quadrature and interpolation was introduced by Smolyak. The basis of this method is the Sparse tensor product. In following, the construction of this method is represented. Suppose that $Q_{l}^{(1)} f$ a family of quadrature rules and will have:

$$
\begin{aligned}
& \Delta_{l}^{(1)} f \equiv\left(Q_{l}^{(1)}-Q_{l-1}^{(1)}\right) f \\
& Q_{0}^{(1)} f \equiv 0 .
\end{aligned}
$$

Note that $\Delta_{l}^{(1)} f$ is also a quadrature rule. For nested formulas, $\Delta_{l}^{(1)} f$ contains the set of nodes of $Q_{l}^{(1)} f$ with weights equal to the difference of weights between level $l$ and $l-1$.

The sparse cubature can construct by the multi-index 1 $=\left(l_{1}, \ldots, l_{N}\right) \in \mathrm{N}^{N}$ and define

$|\mathbf{l}| \equiv \sum_{i=1}^{N} l_{i}$

this multi-index at level $l$ is used and then the sparse cubature formula is shown as

$$
Q_{l}^{(N)} f \equiv \sum_{|1| \leq l+N-1}\left(\Delta_{l_{1}}^{(1)} \otimes \cdots \otimes \Delta_{l_{N}}^{(1)}\right) f
$$

where the support nodes expressed in multi-index as $|\mathbf{I}|$, $\left(|\mathbf{I}|=l_{1}+\ldots+l_{d}\right)$. The dimension of function $f$ is shown by $N$. The interpolant expressed by using a recursive manner as follows (Eq. (21)):

$$
Q_{l}^{(d)} f=\sum_{l+1 \leq 1 \mid \leq l+d}(-1)^{l+d-|\mathbf{k}|}\left(\begin{array}{c}
d-1 \\
l+d-|\mathbf{k}|
\end{array}\right) \quad\left(Q_{k_{1}}^{(1)} \otimes \cdots \otimes Q_{k_{d}}^{(1)}\right) f .
$$

With the Smolyak algorithm, the weight $w_{i}$ corresponding to the $i$ th collocation point $\xi_{i}$ is defined,

$w_{i}=(-1)^{l+d-|\mathbf{k}|}\left(\begin{array}{c}d-1 \\ l+d-|\mathbf{k}|\end{array}\right)\left(w_{k_{1}}^{i_{1}} \otimes \cdots \otimes w_{k_{d}}^{i_{d}}\right)$.

Then, by the sparse grid method, the mean and standard deviation of the objective can be computed as
$E[f]=\sum_{k} w_{k} f\left(\xi_{k}\right)$

$\operatorname{Var}[f]=E[f]-(E[f])^{2}$.

\subsection{Adaptive grid refinement}

In the conventional sparse grid collocation method, the same divisions in all are considered. While, the final solution is not dependent to some of these areas. As a result, the distribution of collocation points should change in a smart way.

For clarity, first a list of definitions for frequently used terms is given here. Most of these definitions are taken from [22]:

- A multi-index represents one combination of $1 \mathrm{D}$ quadrature rules to form a $\mathrm{N}$-dimensional grid component. For example: multi-index $(1,3)$ would represent a 2-dimensional grid component formed by the tensor product $Q_{1} \otimes Q_{2}$.

- An index set is a set of multi-indices that represent the components of the sparse grid. An index set X is admissible if it satisfies the following conditions for generalized sparse grid construction for all multiindices $\mathrm{k} \in \mathrm{X}$ :

$\mathrm{k}-\mathbf{e}_{\mathbf{j}} \in \mathrm{X}$, for $1 \leq j \leq d, \quad k_{j}>1$,

where $\mathbf{k}$ is a multi-index, $\mathbf{e}_{\mathbf{j}}$ is the $j$-th unit vector and $d$ is the number of dimensions of the problem. In other words, any admissible index set for each k contains all indices which have smaller entries than $\mathbf{k}$ in at least one dimension.

- The forward neighbors of a multi-index $k$ is defined as the $d$ multi-indices $\left\{\mathrm{k}+\mathbf{e}_{\mathrm{j}}, 1 \leq j \leq N\right\}$. By extension, the forward neighbors of a grid are all multi-indices which satisfy this definition, and are not yet part of the index set X. As an example, consider a level 2, 2-dimensional sparse grid. The index set for this grid is: $\{(1,1),(2,1),(1,2)\}$. The set of forward neighbors for this grid would be: $\{(3,1),(2,2),(1,3)\}$. This is depicted in Fig. 1. The multi-indices of the sparse grid are shown in dark gray, while the three forward neighbors are light gray.
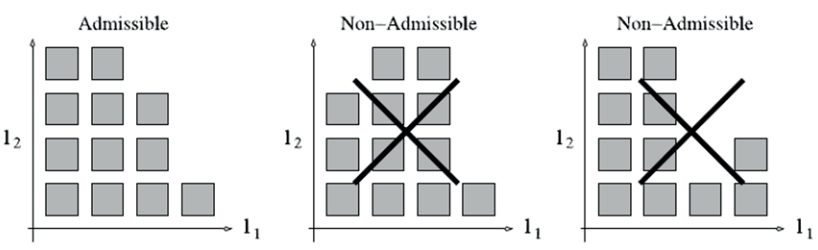

Fig. 1 Diagram showing the forward neighbors of a level 2, 2-dimensional sparse grid 
- The backward neighbors of a multi-index are defined as all multi-indices $\left\{\mathrm{k}-\mathbf{e}_{\mathbf{j}}, 1 \leq j \leq N\right\}$.

- A parent interaction is an interaction which contains exactly $(i-1)$ of the indices of the current interaction, where $i$ indicates the order of the current interaction. As an example, for third order interaction $(1,2,4)$ there are exactly three parent interactions: $(1,2),(1,4)$ and $(2,4)$.

The Gerstner and Griebel adaptive approach is explained in detail in [23], which the information contained in this section is based on. This approach divides the index set $X$ into two disjoint sets, called active and old index set. The active index set $A$ contains the forward neighbors of the old index set $X$. For each of the multi-indices in A, an error indicator is calculated:

$g_{j}=\left|I_{X}-I_{X+A_{j}}\right|$,

where $g$ is the error indicator, $I$ indicates the computed value of the integral and $j$ indicates a multi-index in $A$. In other words, the error indicator calculates the difference between the calculated value of the integral with the old index set, the value of the integral calculated with the old index set plus one of the multi-indices in the active index set. This is done for each entry in $A$. The global error estimate $\lambda$ is then the sum of all $g_{j}$ :

$\lambda=\sum_{j=1}^{m} g_{j}$

where $m$ is the number of multi-indices in $A$. For each iterative refinement step, the following actions are taken:

- The index with the largest associated error indicator is selected from the active index set and put into the old index set.

- Its associated error estimate is subtracted from the global error estimate $\lambda$.

- At the same time the new admissible forward neighbors are added to the active index set. The global error estimate is recalculated by determining the error estimators of these new forward neighbors.

- The value of the integral is updated.

- If the global error estimate falls below a given threshold, the computation is stopped and the computed integral value is returned. If this is not the case, the cycle goes back to the first step.

\section{Optimization algorithm}

The optimization algorithm using of the Adaptive Sparse Grid Collocation methods can be written as follows:
1. Problem discretize and initialization progress was preformed.

2. Stochastic field discretization KL by Eq. (14).

3. $M$ integration points and the corresponding weights using the adaptive sparse grid method Generated.

4. Optimization loop preformed until the convergence criterion is satisfied:

- For $k=1, \ldots, M$ computes $\mathbf{K}_{k} \mathbf{u}_{k}=\mathbf{f}_{k}$ and $\partial C_{k} / \partial \rho$.

- The mean and the variance Estimated from Eq. (23)

- The mean and variance sensitivities with respect to design variable evaluated

- The robust objective sensitivities computed from Eq. (12)

- Update $\rho$.

The mean and the variance gradients are computed based on the gradients of the samples. For each sample the standard adjoint sensitivity analysis is used for estimating the sensitivities of the objective $\partial C_{k} / \partial \rho$ and the constraints.

Keeping the solution vectors $\mathbf{u}_{k}$ can become very expensive in terms of memory. Their contribution to the expectation and the variance, as well as to the sensitivities, can be added during the loop through the collocation points.

\section{Numerical examples}

The presented methodology is demonstrated in the design of a 2D cantilever and a MBB beam. The results are obtained with a modulus of elasticity, $\mathrm{E}=1.0$, a penalization parameter $p=3$, and $\mathrm{E}_{\min }=10^{-4}$. All optimizations start with $\beta=1$, and the projection parameter is doubling every 50 iterations. The final projection parameter is $\beta=10$. Control parameters in Eq. (16), have been considered as $\alpha=0.5$ and $\alpha=0.25$. The design variables are updated using optimality criteria $(\mathrm{OC})$ method. The optimization process is terminated

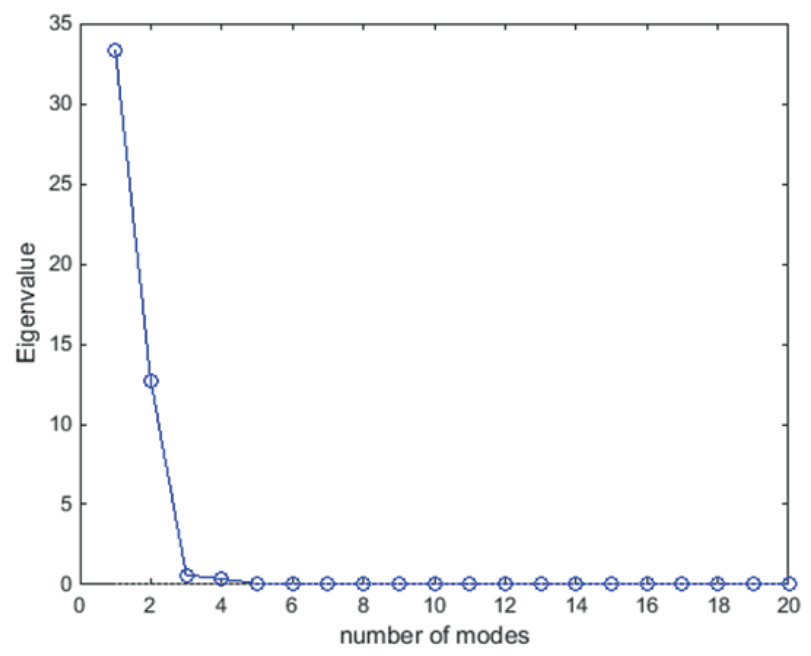

Fig. 2 Correlation eigenvalues of random field $\mathrm{Z}$ 
when the largest change in the design variables becomes smaller than $1 \%$. The topology optimization process is performed using MATLAB software [24]. The first 100 eigenvalues of the correlation matrix $R$ over a uniform mesh are shown in Fig. 2, where their fast modal decay is apparent.

In practice, firstly truncate (KL) to $N$ terms and then used the ratio $\left(\sum_{i=1}^{n_{\text {mode }}} \lambda_{i}\right) /\left(\sum_{i=1}^{n} \lambda_{i}\right)$ to check the sufficiency of the number of truncated modes which indicates the first $n_{\text {mode }}$ modes to represent the random field. This measure for $n_{\text {mode }}=4$ is 0.9566 , i.e. this truncation yields a $96 \%$ $Z$ representation which deem sufficient.

\subsection{D Simply supported beam}

As the first example, the design domain, the boundary, and loading conditions of the simply supported beam are illustrated in Fig. 3. The beam dimensions are considered as $L=90 \mathrm{~mm}$ and $H=30 \mathrm{~mm}$ and the thickness is $T=1 \mathrm{~mm}$. The material is assumed to have $E_{0}=1 \mathrm{MPa}$ and Poisson's ratio of 0.30. A single random load case is considered with which the angle and magnitude are characterized by two independent random variables. The angle is assumed to follow a uniform distribution with the interval of $[-3 \pi / 4,-\pi / 4]$. The Gaussian distribution of the magnitude has a mean of 1 and standard deviation of 0.3 . The goal of robust optimization is to minimize the compliance of the structure means that optimizing the mean $\mu_{f}$ and minimizing standard deviation $\sigma_{f}$ of under the volume constraint. The weight $\kappa$ is set to be 1 in this example. For discretization of the design space, a mesh size that equal with $90 * 30$ of four-node quadrilateral elements has been used.

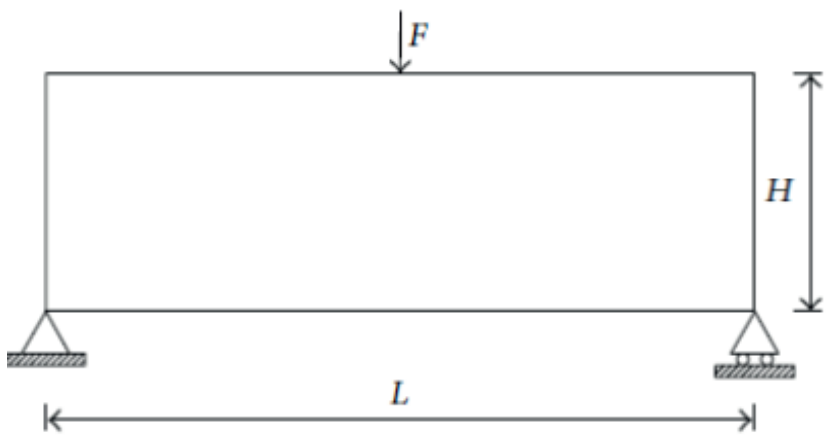

Fig. 3 Design domain and boundary conditions of a 2D simply supported beam

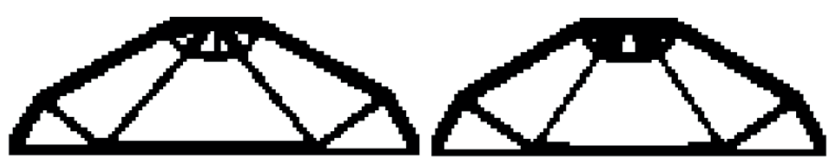

Fig. 4 Design obtained for deterministic, Obj. $=35.6935$, (right) and robust design optimization $(\mathrm{RDO}), \mathrm{Obj} .=25.4565,(\mathrm{left})$
Table 1 Comparison of robust design of different methods

\begin{tabular}{lcccc}
\hline Method & Mean & STD & Objective & Time \\
\hline ASGC & 20.44 & 5.57 & 26.01 & $55 \mathrm{~s}$ \\
Monte Carlo & 21.37 & 11.13 & 32.5 & $302 \mathrm{~s}$ \\
Tensor product grid & 22.26 & 11.85 & 34.11 & $59 \mathrm{~s}$ \\
Smolyak Sparse grid & 22.23 & 11.84 & 34.07 & $59 \mathrm{~s}$ \\
\hline
\end{tabular}

The optimal topologies obtained by deterministic and the proposed robust topology optimization approaches are presented in Fig. 4.

The DTO and RTO resulted in significantly different topologies, as shown in Fig. 4. The remarkable difference lies in the robust design exhibiting an asymmetric layout compared with the deterministic design characterized by symmetric configuration. It can be attributed to the fact that the deterministic design suffers from symmetric vertical constraints at the lower left and the lower right corner. However, in robust design, the structure possesses an asymmetrical degree of freedom constraint in horizontal direction under the condition of the horizontal component of the load. As the results showed, the robust designs present more robust topologies than the deterministic approach, according to the results summary of the statistical information. This emphasizes the importance of considering the uncertainties in load for structural design. It is also noted that the corresponding computation cost for robust designs is larger than for the deterministic design since multiple load cases are required for calculating the mean and deviation values of the performance function.

The optimal topologies obtained by different robust topology optimization approaches results are compared in Table 1.

The results of Monte Carlo simulations in Table 1 show that the proposed robust approach possesses improved efficiency without losing much accuracy. At the same time, compared with sparse grid, the computation burden for the exponential dependent calculation of tensor product grid can be overcome to a certain extent. This means sparse grid, in a manner, performs better than the tensor product grid in compromise between robustness and cost in robust design. Finally by comparison standard deviation of these methods can conclude ASGC method is better than other methods because in topology optimization final goal is minimize the standard deviation. By minimizing it the design topology has a stable behavior.

From the comparison of the results, with the improvement of level $l$ accuracy, the number of collocation points in sparse grid corresponding increases. As the results listed 
in Table 2 show, the mean and standard deviation of the compliance for sparse grid are gradually decreasing with increase of level $l$. This indicates that more robust designs are clearly obtained under higher level $l$. Meanwhile, the higher computation cost is required due to the increase in the number of evaluations of performance function responses. This means robustness enhancement is inevitably accompanied by the sacrifice of convergence time.

\subsection{Robust design of a cantilever beam}

The design domain, the boundary conditions for the state problem are shown in Fig. 5. The simulations are performed with beam height $\mathrm{L}=1$. The applied force is $\mathrm{F}=0.1$. The volume fraction of the solid material is set to $50 \%$ of the total volume.

Optimized designs for a single uniformly distributed threshold $\eta \in U[0.3,0.7]$ are shown in Fig. 6. The designs do not possess features comparable with the mesh size. The designs are obtained with deterministic and RDO are shown in Fig. 6 and the results are listed in Table 3.

When the weight of the standard deviation, $\kappa$, is small, its increase does not have a significant effect on the objective function (compliance), because its contribution to the robust objective (9) is small. The results of the increasing in $\kappa$ are denoted in Table 4 for $\eta \in U[0.3,0.7]$.

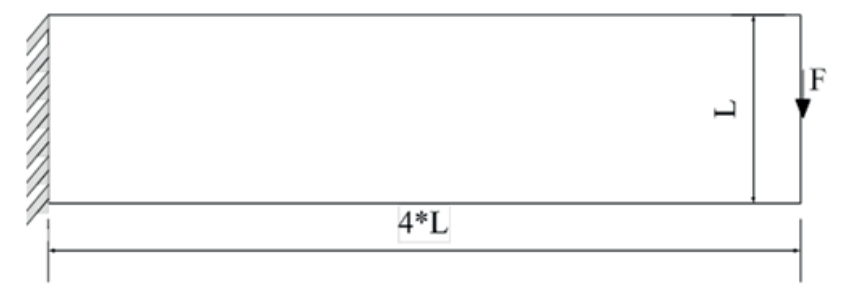

Fig. 5 Design domain and boundary condition for cantilever beam

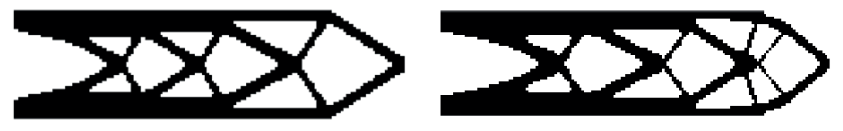

Fig. 6 Design obtained for deterministic (right) and robust design optimization (RDO)(left)
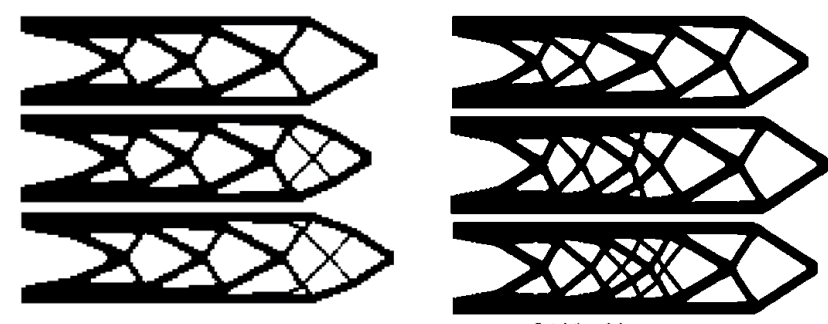

Ascc methos

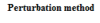

Fig. 7 Robust designs obtained with $\kappa=1$ (top), $\kappa=3, \kappa=5$ (bottom)
Table 2 Comparison of robust design of different level 1

\begin{tabular}{lccc}
\hline Method & Mean & STD & Number of points \\
\hline Sparse grid $(l=1)$ & 20.44 & 5.57 & 5 \\
Sparse grid $(l=2)$ & 20.13 & 5.43 & 13 \\
Sparse grid $(l=3)$ & 19.73 & 5.01 & 29 \\
\hline
\end{tabular}

Table 3 Minimum Compliance of deterministic and robust design

\begin{tabular}{lc}
\hline Compliance & Method \\
\hline 3.7354 & Deterministic. \\
3.6157 & RDO $(\kappa=1)$ \\
\hline
\end{tabular}

Table 4 The effect of increasing in $\kappa$ statistical moment and Compliance

\begin{tabular}{lccc}
\hline STD & Mean & Objective & $\kappa$ \\
\hline 0.2920 & 3.2508 & 3.6157 & $\kappa=1$ \\
0.2859 & 3.1869 & 4.2591 & $\kappa=3$ \\
0.2803 & 3.1453 & 5.0499 & $\kappa=5$ \\
\hline
\end{tabular}

Table 5 Comparison of robust design of different methods

\begin{tabular}{lccc}
\hline ASGC & Perturbation & Mont Carlo & $\kappa$ \\
\hline 3.2508 & 3.59 & 3.58 & $\kappa=1$ \\
3.1869 & 3.67 & 3.60 & $\kappa=3$ \\
3.1453 & 3.83 & 3.62 & $\kappa=5$ \\
\hline
\end{tabular}

Table 6 The effect of increasing the threshold interval for $\kappa=3$

\begin{tabular}{lcccc}
\hline \multicolumn{2}{l}{ Present work(ASGC) } & \multicolumn{3}{l}{ Monte Carlo method } \\
Mean & STD & Mean & STD & Interval \\
\hline 3.1805 & 0.2843 & 3.69 & 0.42 & {$[0.1,0.9]$} \\
3.1881 & 0.2857 & 3.70 & 0.59 & {$[0.2,0.8]$} \\
3.1869 & 0.2859 & 3.70 & 0.51 & {$[0.3,0.7]$} \\
3.2465 & 0.2918 & 3.74 & 0.66 & {$[0.4,0.6]$} \\
\hline
\end{tabular}

Increasing the weight $\kappa$ in the objective given in Eq. (9), decreases the mean compliance and decreases its variation. Therefore, the mechanism response becomes more robust with respect to variations in the geometric variation.

To validate the present method, a comparison has been done between this method with Perturbation method (Lazarov work). The results of this comparison are shown in Fig. 7 and the minimum compliance values obtained from these methods are denoted in Table 5. RDO results related to three designs with weight parameter $\kappa=1, \kappa=3$, and $\kappa=5$.

From the results of Table 5 can be seen that the mean compliance of structure decreased with increasing in $\kappa$. Therefore, these methods have the same response. But, from Fig. 7 can be concluded the ASGC method has a better answer compared with the other two methods. It can be seen that when the weight parameter $\kappa$ increased, in perturbation method inside of the structure changed significantly 
but in the ASGC method this changed smoothly occurred. This means that the structure becomes more robust with respect to variations in the geometric variation.

Results of increasing the threshold interval are denoted in Table 6 for $\kappa=3$. As expected, the standard deviation is decreasing with expanding the support domain of the threshold distribution this means that, a larger threshold interval leads to a more robust behavior. Therefore, expanding threshold interval has a similar effect with increasing in $\kappa$.

\section{Conclusions}

A systematic approach for topology optimization under uncertainty is introduced that relies on the Adaptive Sparse Grid Collocation methods (ASGC) for uncertainty propagation of the cost and constraint functions. The expressions of the stochastic objective and its sensitivities are derived, and the main computational steps are presented in details. Different numerical examples for topology optimization under uncertainty are considered. The computation of the random threshold field is elucidated in the numerical examples. In these examples, uncertainty is modeled by using a random threshold field that is characterized by a truncated Karhunen-Loeve expansion.

\section{References}

[1] Bendsøe, M. P., Sigmund, O. "Topology optimization, Theory, Methods, and Applications", $1^{\text {st }}$ ed., Springer, Berlin, Germany, 2004. https://doi.org/10.1007/978-3-662-05086-6

[2] Ben-Tal, A., Nemirovski, A. "Robust truss topology design via semidefinite programming", SIAM Journal on Optimization, 7(4), pp. 991-1016, 1997.

https://doi.org/10.1137/S1052623495291951

[3] Guest, J. K., Igusa, T. "Structural optimization under uncertain loads and nodal locations", Computer Methods in Applied Mechanics and Engineering, 198(1), pp. 116-124, 2008.

https://doi.org/10.1016/j.cma.2008.04.009

[4] Kogiso, N., Ahn, W., Nishiwaki, S., Izui, K., Yoshimura, M. "Robust Topology Optimization for Compliant Mechanisms Considering Uncertainty of Applied Loads", Journal of Advanced Mechanical Design, Systems, and Manufacturing, 2(1), pp. 96-107, 2008. https://doi.org/10.1299/jamdsm.2.96

[5] Dunning, P. D., Kim, H. A., Mullineux, G. "Introducing Loading Uncertainty in Topology Optimization", AIAAJ, 49(4), pp. 760$768,2011$.

https://doi.org/10.2514/1.J050670

[6] Cherkaev, E., Cherkaev, A. "Minimax optimization problem of structural design", Computers and Structures, 86(13-14), pp. 14261435, 2008.

https://doi.org/10.1016/j.compstruc.2007.05.026
In the case of random load by comparison of different method that listed in Table 2 can conclude ASGC have a better behavior. Advantages of this method can be summarized as minimum time, and then less cost, less standard deviation, minimum compliance in comparison with other method.

Comparing ASGC with some method such as Monte Carlo, Perturbation for geometry uncertainty is demonstrated that the computational burden of ASGC is smaller than the above mentioned methods. For a single random variable, the presented approach is faster than optimization based on sampling method based on Monte Carlo with $10^{5}$ realizations.

It is also shown that the optimum volume and minimum compliance obtained by using this method is smaller than other method. As well as the effect of increasing of weight parameter, $\kappa$, was discussed and showed by increasing this parameter the structures become robust and mean and standard deviation of compliance are decreased. It was shown that in comparing ASGC method and Smolyak Sparse grid, however, both methods are used of sparse grid method, but ASGC automatically detects which dimensions require more nodal points and not treats all dimensions equally.

[7] Lógó, J. "New Type of Optimality Criteria Method in Case of Probabilistic Loading Conditions", Mechanics Based Design of Structures and Machines, 35(2), pp. 147-162, 2007. https://doi.org/10.1080/15397730701243066

[8] Lógó, J., Ghaemi, M., Rad, M. M. "Optimal Topologies in Case of Probabilistic Loading: The Influence of Load Correlation", Mechanics Based Design of Structures and Machines, 37(3), pp. 327-348, 2009. https://doi.org/10.1080/15397730902936328

[9] Zhao, J., Wang, C. "Robust Topology Optimization of Structures Under Loading Uncertainty", AIAAJ, 52(2), pp. 398-407, 2014. https://doi.org/10.2514/1.J052544

[10] Zhao, Q., Chen, X., Ma, Z.-D., Lin, Y. "Robust Topology Optimization Based on Stochastic Collocation Methods under Loading Uncertainties", Mathematical Problems in Engineering, 2015, Article ID 580980, 2015. https://doi.org/10.1155/2015/580980

[11] Chen, S., Chen, W., Lee, S. "Level set based robust shape and topology optimization under random field uncertainties", Structural and Multidisciplinary Optimization, 41(4), pp. 507-524, 2010. https://doi.org/10.1007/s00158-009-0449-2

[12] Tootkaboni, M., Asadpoure, A., Guest, J. K. "Topology optimization of continuum structures under uncertainty - A Polynomial Chaos approach", Computer Methods in Applied Mechanics and Engineering, 201-204, pp. 263-275, 2012. https://doi.org/10.1016/j.cma.2011.09.009 
[13] Chen, S., Chen, W. "A new level-set based approach to shape and topology optimization under geometric uncertainty", Structural and Multidisciplinary Optimization, 44(1), pp. 1-18, 2011.

https://doi.org/10.1007/s00158-011-0660-9

[14] Allaire, G., Dapogny, C. "A linearized approach to worst-case design in parametric and geometric shape optimization", Mathematical Models and Methods in Applied Sciences, 24(11), pp. 2199-2257, 2014 .

https://doi.org/10.1142/S0218202514500195

[15] Lazarov, B. S., Schevenels, M., Sigmund, O. "Topology optimization considering material and geometric uncertainties using stochastic collocation methods", Structural and Multidisciplinary Optimization, 46(4), pp. 597-612, 2012.

https://doi.org/10.1007/s00158-012-0791-7

[16] Zhou, M., Lazarov, B. S., Sigmund, O. "Topology optimization for optical projection lithography with manufacturing uncertainties", Applied Optics, 53(12), pp. 2720-2729, 2014. https://doi.org/10.1364/AO.53.002720

[17] Lazarov, B. S., Schevenels, M., Sigmund, O. "Topology optimization with geometric uncertainties by perturbation techniques", International Journal for Numerical Methods in Engineering, 90(11), pp. 1321-1336, 2012. https://doi.org/10.1002/nme.3361

[18] Sigmund, O. "Manufacturing tolerant topology optimization", Acta Mechanica Sinica, 25(2), pp. 227-239, 2009. https://doi.org/10.1007/s10409-009-0240-z
[19] Keshavarzzadeh, V., Fernandez, F., Tortorelli, D. A. "Topology optimization under uncertainty via non-intrusive polynomial chaos expansion", Computer Methods in Applied Mechanics and Engineering, 318(1), pp. 120-147, 2017. https://doi.org/10.1016/j.cma.2017.01.019

[20] Latifi Rostami, S. A., Ghoddosian, A. "Topology optimization of continuum structures under hybrid uncertainties", Structural and Multidisciplinary Optimization, 57(6), pp. 2399-2409, 2018. https://doi.org/10.1007/s00158-017-1868-0

[21] Bruns, T. E., Tortorelli, D. A. "Topology optimization of non-linear elastic structures and compliant mechanisms", Computer Methods in Applied Mechanics and Engineering, 190(26-27), pp. 34433459, 2001. https://doi.org/10.1016/S0045-7825(00)00278-4

[22] Schevenels, M., Lazarov, B. S., Sigmund, O. "Robust topology optimization accounting for spatially varying manufacturing errors", Computer Methods in Applied Mechanics and Engineering, 200(49-52), pp. 3613-3627, 2011. https://doi.org/10.1016/j.cma.2011.08.006

[23] Gerstner, T., Griebel, M. "Dimension-Adaptive Tensor-Product Quadrature", Computing, 71(1), pp. 65-87, 2003. https://doi.org/10.1007/s00607-003-0015-5

[24] MathWorks "MATLAB Version 8.5 (R2015a)", [computer program] 